\title{
New Generalization of $f$-Best Simultaneous Approximation in Topological Vector Spaces
}

\author{
Mahmoud Rawashdeh and Sarah Khalil \\ Department of Mathematics and Statistics, Jordan University of Science and Technology, Irbid 22110, Jordan \\ Correspondence should be addressed to Mahmoud Rawashdeh; msalrawashdeh@just.edu.jo
}

Received 20 January 2013; Revised 23 April 2013; Accepted 23 April 2013

Academic Editor: Naseer Shahzad

Copyright ( 2013 M. Rawashdeh and S. Khalil. This is an open access article distributed under the Creative Commons Attribution License, which permits unrestricted use, distribution, and reproduction in any medium, provided the original work is properly cited.

\begin{abstract}
Let $K$ be a nonempty subset of a Hausdorff topological vector space $X$, and let $f$ be a real-valued continuous function on $X$. If for each $x=\left(x_{1}, x_{2}, \ldots, x_{n}\right) \in X^{n}$, there exists $k_{0} \in K$ such that $F_{K}(x)=\sum_{i=1}^{n} f\left(x_{i}-k_{0}\right)=\inf \left\{\sum_{i=1}^{n} f\left(x_{i}-k\right): k \in K\right\}$, then $K$ is called $f$-simultaneously proximal and $k_{0}$ is called $f$-best simultaneous approximation for $x$ in $K$. In this paper, we study the problem of $f$-simultaneous approximation for a vector subspace $K$ in $X$. Some other results regarding $f$-simultaneous approximation in quotient space are presented.
\end{abstract}

\section{Introduction}

Let $K$ be a closed subset of a Hausdorff topological vector space $X$ and $f$ a real-valued continuous function on $X$. For $x \in X$, set $F_{K}(x)=\inf _{k \in K} f(x-k)$. A point $k_{0} \in K$ is called $f$-best approximation to $x$ in $K$ if $F_{K}(x)=f\left(x-k_{0}\right)$. The set $P_{K}^{f}(x)=\left\{k \in K: F_{K}(x)=f(x-\kappa)\right\}$ denotes the set of all $f$-best approximations to $x$ in $K$. Note that this set may be empty. The set $K$ is said to be $f$-proximal ( $f$-Chebyshev) if for each $x \in X, P_{K}^{f}(x)$ is nonempty (singleton). The notion of $f$-best approximation in a vector space $X$ was given by Breckner and Brosowski [1] and in a Hausdorff topological space $X$ by Narang $[2,3]$. For a Hausdorff locally convex topological vector space and a continuous sublinear functional $f$ on $X$, certain results on best approximation relative to the functional $f$ were proved in $[1,4]$. By using the existence of elements of $f$-best approximation, certain results on fixed points were proved by Pai and Veermani in [5]. In addition, for a topological vector space $X$ relative to upper semicontinuous functions, some results on best approximation were proved by Haddadi and Hamzenejad [6]. Moreover, Naidu [7] proved some results on best simultaneous approximation related to $f$-nearest point and topological vector space $X$.
Analogous to the problem of simultaneous approximation [8], we introduce the concept of best $f$-simultaneous approximation as follows.

Definition 1. Let $K$ be a non-empty subset of a Hausdorff topological vector space $X$, and let $f$ be a real-valued continuous function on $X$. A point $k_{0} \in K$ is called $f$ best simultaneous approximation in $K$ if there exists $x=$ $\left(x_{1}, x_{2}, \ldots, x_{n}\right) \in X^{n}$ such that

$$
F_{K}(x)=\inf \left\{\sum_{i=1}^{n} f\left(x_{i}-k\right): k \in K\right\}=\sum_{i=1}^{n} f\left(x_{i}-k_{0}\right) .
$$

The set of all $f$-best simultaneous approximations to $x=$ $\left(x_{1}, x_{2}, \ldots, x_{n}\right) \in X^{n}$ in $K$ is denoted by

$$
P_{K}^{f}(x)=\left\{k \in K: F_{K}(x)=\sum_{i=1}^{n} f\left(x_{i}-k\right)\right\} .
$$

The set $K$ is called $f$-simultaneously proximal ( $f$-simultaneously Chebyshev) if for each $x=\left(x_{1}, x_{2}, \ldots, x_{n}\right) \in X^{n}$, $P_{K}^{f}(x) \neq \phi$ (singleton). If $n=1$, simultaneous $f$-proximal is precisely $f$-proximal. 
We remark that if $f(x)=\|x\|$, then the concept of $f$-best approximation is precisely the best approximation.

A set $K$ is said to be inf-compact at a point $x=$ $\left(x_{1}, x_{2}, \ldots, x_{n}\right) \in X^{n}[5]$ if each minimizing sequence in $K$ (i.e., $\left.\sum_{i=1}^{n} f\left(x_{i}-k_{n}\right) \rightarrow F_{K}(x)\right)$ has a convergent subsequence in $K$. The set $K$ is called inf-compact if it is inf-compact at each $x=\left(x_{1}, x_{2}, \ldots, x_{n}\right) \in X^{n}$.

It is easy to see that if $K$ is compact or inf-compact, then $K$ is $f$-simultaneously proximal.

In this paper, we introduce the concept of $f$-simultaneous approximation and study the existence and uniqueness problem of $f$-simultaneous approximation of a subspace $K$ of a Hausdorff topological vector space $X$. Certain results regarding $f$-simultaneous approximation in quotient spaces are obtained by generalizing some of the results in [9].

Throughout this paper, $X$ is a Hausdorff topological vector space and $f$ is a real-valued continuous function on $X$.

\section{2. $f$-Simultaneous Approximation}

In this section, we give some characterizations of $f$-proximal sets in $X$. We begin with the following definitions.

Definition 2. A function $f: X \rightarrow \mathbb{R}$ is called absolutely homogeneous if $f(\alpha x)=|\alpha| f(x)$, for all $x \in X$ and all $\alpha \in \mathbb{R}$.

Definition 3. A subset $K$ of $X$ is called $f$-closed if for all sequences $\left\{k_{m}\right\}$ of $K$ and for all $x=\left(x_{1}, x_{2}, \ldots, x_{n}\right) \in X^{n}$, such that $\sum_{i=1}^{n} f\left(x_{i}-k_{m}\right) \rightarrow 0$, we have $x \in K^{n}$.

Definition 4. A subset $K$ of $X$ is called $f$-compact if for every sequence $\left\{k_{n}\right\}$ in $K$ there exist a subsequence $\left\{k_{n_{k}}\right\}$ of $\left\{k_{n}\right\}$ and $k_{0} \in K$ such that $f\left(k_{n_{k}}-k_{0}\right) \rightarrow 0$.

Definition 5. For $x, y \in X$, where $x=\left(x_{1}, x_{2}, \ldots, x_{n}\right) \in X^{n}$ and $y=\left(y_{1}, y_{2}, \ldots, y_{n}\right) \in X^{n}, x$ is said to be $f$-orthogonal to $y$ denoted by $x \perp_{f} y$, if $\sum_{i=1}^{n} f\left(x_{i}\right) \leq \sum_{i=1}^{n} f\left(x_{i}+\alpha y_{i}\right)$ for every scalar $\alpha \in \mathbb{R}$. Also, $x$ is said to be $f$-orthogonal to a set $K$ if $x \perp_{f} k$, for all $k \in K$.

Definition 6. We say that $K$ is $w$-compact if every net $\left\{k_{\alpha}\right\}$ in $K$ has a convergent subnet.

Theorem 7. Let $K$ be a subset of $X$. Then, one has the following.

(1) $F_{K+y}(x+Y)=F_{K}(x)$, for all $x=\left(x_{1}, x_{2}, \ldots, x_{n}\right)$, where $Y=(y, y, \ldots, y) \in X^{n}$.

(2) $P_{K+y}^{f}(x+Y)=P_{K}^{f}(x)+y$, for all $x=\left(x_{1}, x_{2}, \ldots, x_{n}\right)$.

(3) $K$ is $f$-simultaneously proximal ( $f$-simultaneously Chebyshev) if and only if $K+y$ is $f$-simultaneously proximal ( $f$-simultaneously Chebyshev) for every $y \in$ $X$.

Moreover, if $f$ is absolutely homogeneous function, then one has the following.

(4) $F_{\alpha K}(\alpha x)=|\alpha| F_{K}(x)$, for all $x=\left(x_{1}, x_{2}, \ldots, x_{n}\right) \in X^{n}$ and $\alpha \in \mathbb{R}$.
(5) $P_{\alpha K}^{f}(\alpha x)=\alpha P_{K}^{F}(x)$, for all $x=\left(x_{1}, x_{2}, \ldots, x_{n}\right) \in X^{n}$ and $\alpha \in \mathbb{R}$.

(6) $K$ is $f$-simultaneously proximal ( $f$-simultaneously Chebyshev) if and only if $\alpha K$ is $f$-simultaneously proximal ( $f$-simultaneously Chebyshev), $\alpha \in \mathbb{R}$.

(7) If $f$ is convex function and $K$ is a convex set, then $P_{K}^{f}(x)$ is convex.

Proof. (1) Let $x=\left(x_{1}, x_{2}, \ldots, x_{n}\right)$ and $Y=(y, y, \ldots, y) \in X^{n}$. Then

$$
F_{K+y}(x+Y)=\inf _{k \in K} \sum_{i=1}^{n} f\left(\left(x_{i}+y\right)-(\kappa+y)\right)=F_{K}(x) .
$$

(2) The equation

$$
\begin{aligned}
\sum_{i=1}^{n} f\left(x_{i}-k_{0}\right) & =\inf _{k \in K} \sum_{i=1}^{n} f\left(\left(x_{i}+y\right)-(k+y)\right) \\
& =\inf _{k \in K} \sum_{i=1}^{n} f\left(x_{i}-k\right)
\end{aligned}
$$

implies that $k_{0}+y \in P_{K+y}^{f}(x+Y)$ if and only if $k_{0} \in P_{K}^{f}(x)$. Thus,

$$
P_{K+y}^{f}(x+Y)=P_{K}^{f}(x)+y .
$$

(3) The proof follows immediately from part (2) above.

(4) Let $x=\left(x_{1}, x_{2}, \ldots, x_{n}\right) \in X^{n}, \alpha \in \mathbb{R}$. Then,

$$
\begin{aligned}
F_{\alpha K}(\alpha x) & =\inf _{k \in K} \sum_{i=1}^{n} f\left(\alpha x_{i}-\alpha k\right) \\
& =|\alpha| \inf _{k \in K} \sum_{i=1}^{n} f\left(x_{i}-k\right)=|\alpha| F_{K}(x) .
\end{aligned}
$$

(5) If $\alpha=0$, then we are done. If $\alpha \neq 0$ and $k_{0} \in P_{\alpha K}^{f}(\alpha x)$, then $k_{0} \in \alpha K$ and

$$
\sum_{i=1}^{n} f\left(\alpha x_{i}-k_{0}\right)=\inf _{k \in K} \sum_{i=1}^{n} f\left(\alpha x_{i}-\alpha k\right) .
$$

This implies that

$$
\sum_{i=1}^{n} f\left(x_{i}-\frac{1}{\alpha} k_{0}\right)=F_{K}(x),
$$

which implies that $(1 / \alpha) k_{0} \in P_{K}^{f}(x)$.

(6) The proof follows immediately from part (5) above.

(7) Let $k_{1}, k_{2} \in P_{K}^{f}(x)$. Since $K$ is convex, then $\lambda k_{1}-(1-$ $\lambda) k_{2} \in K$. We must show that $\lambda k_{1}-(1-\lambda) k_{2} \in P_{K}^{f}(x)$; that is,

$$
\sum_{i=1}^{n} f\left(x_{i}-\left(\lambda k_{1}-(1-\lambda) k_{2}\right)\right)=\inf _{k \in K} \sum_{i=1}^{n} f\left(x_{i}-k\right) .
$$


So,

$$
\begin{aligned}
& \sum_{i=1}^{n} f\left(x_{i}-\left(\lambda k_{1}-(1-\lambda) k_{2}\right)\right) \\
& \quad=\sum_{i=1}^{n} f\left(\lambda\left(x_{i}-k_{1}\right)+(1-\lambda)\left(x_{i}-k_{2}\right)\right) \\
& =\lambda \sum_{i=1}^{n} f\left(x_{i}-k_{1}\right)+(1-\lambda) \sum_{i=1}^{n} f\left(x_{i}-k_{2}\right) \\
& =\lambda F_{K}(x)+(1-\lambda) F_{K}(x) \\
& =F_{K}(x)=\sum_{i=1}^{n} f\left(x_{i}-k\right),
\end{aligned}
$$

which implies that $P_{K}^{f}(x)$ is convex.

Example 8. Let $X=\mathbb{R}^{2}$ and $K=\left\{\left(x_{1}, x_{2}\right) \in \mathbb{R}^{2}: x_{1}^{2}+x_{2}^{2} \leq 4\right\}$, and let $f(x, y)=x^{2}-y^{2}$. If $z=((0,0),(0,1)) \in X^{2}$, then one can show that $F_{K}(z)=f(0,1 / 2)=-1 / 4$.

Theorem 9. Let $f$ be an absolutely homogeneous real-valued function on $X$ and $M$ a vector subspace of $X$. Then,

(1) $F_{M}(\alpha x)=|\alpha| F_{M}(x)$, for all $x=\left(x_{1}, x_{2}, \ldots, x_{n}\right) \in X^{n}$, $\alpha \in \mathbb{R}-\{0\}$;

(2) $P_{M}^{f}(\alpha x)=\alpha P_{M}^{f}(x)$, for all $x=\left(x_{1}, x_{2}, \ldots, x_{n}\right) \in X^{n}$, $\alpha \in \mathbb{R}-\{0\}$.

Proof. (1) Let $x=\left(x_{1}, x_{2}, \ldots, x_{n}\right)$. Then,

$$
\begin{aligned}
F_{M}(\alpha x) & =\inf _{m \in M} \sum_{i=1}^{n} f\left(\alpha x_{i}-m\right) \\
& =|\alpha| \inf _{m^{\prime} \in M} \sum_{i=1}^{n} f\left(x_{i}-m^{\prime}\right)=|\alpha| F_{M}(x) .
\end{aligned}
$$

(2) Let $m_{0} \in P_{M}^{f}(\alpha x)$. Then,

$$
\sum_{i=1}^{n} f\left(\alpha x_{i}-m_{0}\right)=\inf _{m \in M} \sum_{i=1}^{n} f\left(\alpha x_{i}-m\right)
$$

if and only if

$$
\sum_{i=1}^{n} f\left(x_{i}-\frac{1}{\alpha} m_{0}\right)=\inf _{m^{\prime} \in M} \sum^{n} f\left(x_{i}-m^{\prime}\right)=F_{M}(x),
$$

for all $\alpha \in \mathbb{R}-\{0\}$, which implies that $(1 / \alpha) m_{0} \in P_{M}^{f}(x)$, so, $m_{0} \in \alpha P_{M}^{f}(x)$.

Theorem 10. Let $f$ be a positive real-valued function on $X$ such that $x=0$ if and only if $f(x)=0$. Then, if $K$ is $f$ simultaneously proximal, then $K$ is $f$-closed.

Proof. Since $f$ is a positive function, then $\sum_{i=1}^{n} f\left(x_{i}\right) \geq 0$ for all $x=\left(x_{1}, x_{2}, \ldots, x_{n}\right) \in X^{n}$. Let $\left\{k_{m}\right\}$ be a sequence of $K$ and $x=\left(x_{1}, x_{2}, \ldots, x_{n}\right) \in X^{n}$, such that $\sum_{i=1}^{n} f\left(x_{i}-k_{m}\right) \rightarrow 0$. This implies that

$$
F_{K}(x)=\inf _{k \in K} \sum_{i=1}^{n} f\left(x_{i}-k\right) \leq \sum_{i=1}^{n} f\left(x_{i}-k_{m}\right) \longrightarrow 0 .
$$

Since $K$ is $f$-simultaneously proximal, then there exists $k_{0} \in$ $K$ such that

$$
F_{K}(x)=\sum_{i=1}^{n} f\left(x_{i}-k_{0}\right)=0 .
$$

Hence, for all $i=1,2, \ldots, n, f\left(x_{i}-k_{0}\right)=0$. Using the assumption it follows that $x_{i}-k_{0}=0$, and, hence, $x_{i}=k_{0} \in K$. Consequently, $x \in K^{n}$ and $K$ is $f$-closed.

Theorem 11. Let $X$ be a topological vector space and $K$ a vector subspace of $X$. Suppose that $f$ is continuous function and $K$ is $w$-compact; then, $K$ is $f$-simultaneously proximal.

Proof. Let $x=\left(x_{1}, x_{2}, \ldots, x_{n}\right) \in X^{n}$. Since

$$
F_{K}(x)=\inf \sum_{i=1}^{n} f\left(x_{i}-k\right), \quad \text { where } k \in K,
$$

then, for any constant $\alpha$, there exists $\left\{k_{\alpha}\right\}$ such that

$$
\sum_{i=1}^{n} f\left(x_{i}-k_{\alpha}\right) \leq \sum_{i=1}^{n} f\left(x_{i}-k\right)+\frac{1}{\alpha} .
$$

But $K$ is $w$-compact; then, there exists a subnet $\left\{k_{\alpha_{\beta}}\right\}$ such that $k_{\alpha_{\beta}} \rightarrow k_{0}$. Thus,

$$
x_{i}-k_{\alpha_{\beta}} \longrightarrow x_{i}-k_{0}, \quad \forall i=1,2, \ldots, n .
$$

Since $f$ is continuous, then

$$
\sum_{i=1}^{n} f\left(x_{i}-k_{\alpha_{\beta}}\right) \leq \sum_{i=1}^{n} f\left(x_{i}-k\right)+\frac{1}{\alpha} .
$$

Also,

$$
\begin{aligned}
\sum_{i=1}^{n} f\left(x_{i}-k_{0}\right) & =\liminf \sum_{i=1}^{n} f\left(x_{i}-k_{\alpha_{\beta}}\right) \\
& \leq \sum_{i=1}^{n} f\left(x_{i}-k\right) .
\end{aligned}
$$

Hence, $k_{0} \in P_{K}^{f}(x)$.

For a subset $K$ of $X$, let us define $\widehat{K_{F}}$ to be such that

$$
\widehat{K_{F}}=\left\{x=\left(x_{1}, x_{2}, \ldots, x_{n}\right) \in X^{n}: F_{K}(x)=\sum_{i=1}^{n} f\left(x_{i}\right)\right\} \text {. }
$$

Example 12. Consider $X=\left(\mathbb{R}^{2}\right)^{2}$ and $K=\left\{\left(\left(x_{1}, y_{1}\right),\left(x_{2}\right.\right.\right.$, $\left.\left.y_{2}\right)\right): x_{i}=y_{i}$, for all $\left.i=1,2\right\}$. Let $f(x, y)=x^{2}+y^{2}$; then, one can see that

$$
\widehat{K_{F}}=\left\{\left(\left(x_{1},-x_{1}\right),\left(x_{2},-x_{2}\right)\right)\right\} .
$$


Using the previous definition of $\widehat{K_{F}}$, we prove the following theorem characterizing $f$-simultaneously proximal subspaces.

Theorem 13. Let $K$ be a vector subspace of $X$. Then, $K$ is $f$ simultaneously proximal in $X$ if and only if $X^{n}=D_{k}+\widehat{K_{F}}$, where $D_{K}=\{(k, k, \ldots, k): k \in K\}$.

Proof. Suppose that $X^{n}=D_{k}+\widehat{K_{F}}$. Then, for $x=\left(x_{1}, x_{2}\right.$, $\left.\ldots, x_{n}\right) \in X^{n}$, there exists $k_{1}=\left(k_{0}, k_{0}, \ldots, k_{0}\right) \in D_{K}$ and $y=\left(y_{1}, y_{2}, \ldots, y_{n}\right) \in \widehat{K_{F}}$ such that $x=y+k_{1}$. Hence, $x-k_{1}=$ $y \in \widehat{K_{F}}$, and

$$
F_{K}(y)=F_{K}\left(x-k_{1}\right)=\sum_{i=1}^{n} f\left(x_{i}-k_{0}\right),
$$

and so

$$
\begin{aligned}
\sum_{i=1}^{n} f\left(x_{i}-k_{0}\right) & =\inf _{k \in K} \sum_{i=1}^{n} f\left(x_{i}-k_{0}-k\right) \\
& =\inf _{k^{\prime} \in K} \sum_{i=1}^{n} f\left(x_{i}-k^{\prime}\right)=F_{K}(x) .
\end{aligned}
$$

So, $K$ is $f$-simultaneously proximal.

Conversely, suppose that $K$ is $f$-simultaneously proximal and $x=\left(x_{1}, x_{2}, \ldots, x_{n}\right) \in X^{n}$. Then, there exists $k_{0} \in K$ such that

$$
\begin{aligned}
\sum_{i=1}^{n} f\left(x_{i}-k_{0}\right) & =\inf _{k \in K} \sum_{i=1}^{n} f\left(x_{i}-k\right) \\
& =\inf _{k \in K} \sum_{i=1}^{n} f\left(x_{i}-\left(k^{\prime}+k_{0}\right)\right),
\end{aligned}
$$

where $k=k^{\prime}+k_{0}$. If $k_{1}=\left(k_{0}, k_{0}, \ldots, k_{0}\right) \in D_{K}$, then

$$
\sum_{i=1}^{n} f\left(x_{i}-k_{0}\right)=F_{K}\left(x-k_{1}\right) \text {, }
$$

which implies that $x-k_{1}=k_{2} \in \widehat{K_{F}}$ and $X^{n}=D_{k}+\widehat{K_{F}}$.

Proposition 14. Let $X$ be a topological vector space and $K f$ simultaneous proximal subset of $X$. Then,

(1) $k_{0} \in P_{K}^{f}(x)$ if and only if $x-k_{0} \in \widehat{K_{F}}$;

(2) if $f$ is symmetric (i.e., $f(-x)=f(x)$ for all $x \in X$ ), then $x \in \widehat{K_{F}}$ if and only if $-x \in \widehat{K_{F}}$;

(3) if $x \perp_{F} K$, then $x \in \widehat{K_{F}}$, where $x=\left(x_{1}, x_{2}, \ldots, x_{n}\right)$;

(4) if $x \in \widehat{K_{F}}$ and $\alpha K=K$, then $x \perp_{F} K$, where $x=$ $\left(x_{1}, x_{2}, \ldots, x_{n}\right)$.

Proof. (1) Let $k_{0} \in P_{K}^{f}(x)$ if and only if $\sum_{i=1}^{n} f\left(x_{i}-k_{0}\right)=$ $\inf \left\{\sum_{i=1}^{n} f\left(x_{i}-k\right): k \in K\right\}$.
Thus,

$$
\begin{aligned}
& \sum_{i=1}^{n} f\left(x_{i}-k_{0}\right) \\
& =\inf \left\{\sum_{i=1}^{n} f\left(x_{i}-k_{0}+k_{0}-k\right): k \in K\right\} \\
& =\inf \left\{\sum_{i=1}^{n} f\left(x_{i}-k_{0}-k^{\prime}\right): k^{\prime} \in K\right\},
\end{aligned}
$$

which implies that $x-k_{0} \in \widehat{K_{F}}$. then

(2) Let $x=\left(x_{1}, x_{2}, \ldots, x_{n}\right) \in \widehat{K_{F}}$. Since $f$ is symmetric,

$$
\begin{aligned}
\sum_{i=1}^{n} f\left(-x_{i}\right) & =\sum_{i=1}^{n} f\left(x_{i}\right) \\
& =\inf \left\{\sum_{i=1}^{n} f\left(x_{i}-k\right): k \in K\right\} \\
& =\inf \left\{\sum_{i=1}^{n} f\left(-\left(-x_{i}+k\right)\right):-k \in K\right\} \\
& =\inf \left\{\sum_{i=1}^{n} f\left(-x_{i}+k\right):-k \in K\right\} .
\end{aligned}
$$

Hence, $\sum_{i=1}^{n} f\left(-x_{i}\right)=\inf \left\{\sum_{i=1}^{n} f\left(-x_{i}+k\right):-k \in K\right\}$, which implies that

$$
-x=\left(-x_{1},-x_{2}, \ldots,-x_{n}\right) \in \widehat{K_{F}} .
$$

(3) Let $x=\left(x_{1}, x_{2}, \ldots, x_{n}\right)$. Since $x \perp_{F} K$, then

$$
\begin{aligned}
\sum_{i=1}^{n} f\left(x_{i}\right) & \leq \sum_{i=1}^{n} f\left(x_{i}+\alpha k\right) \quad \forall \alpha \in \mathbb{R}, k \in K . \\
& =\sum_{i=1}^{n} f\left(x_{i}-(-\alpha k)\right) \quad \forall \alpha \in \mathbb{R}, k \in K . \\
& =\sum_{i=1}^{n} f\left(x_{i}-k^{\prime}\right), \quad k^{\prime} \in K .
\end{aligned}
$$

So,

$$
\sum_{i=1}^{n} f\left(x_{i}\right) \leq \sum_{i=1}^{n} f\left(x_{i}-k^{\prime}\right), \quad k^{\prime} \in K .
$$

Hence, $x=\left(x_{1}, x_{2}, \ldots, x_{n}\right) \in \widehat{K_{F}}$.

(4) Let $x \in \widehat{K_{F}}$ and $\alpha K=K$. Then,

$$
\begin{aligned}
\sum_{i=1}^{n} f\left(x_{i}\right) & =\inf _{k \in K} \sum_{i=1}^{n} f\left(x_{i}-k\right) \\
& =\inf _{\alpha k \in K} \sum_{i=1}^{n} f\left(x_{i}-\alpha k\right), \quad \text { since } \alpha K=K, \\
& =\inf \sum_{i=1}^{n} f\left(x_{i}+(-\alpha k)\right), \quad \forall k \in K .
\end{aligned}
$$


Thus,

$$
\sum_{i=1}^{n} f\left(x_{i}\right) \leq \sum_{i=1}^{n} f\left(x_{i}+\alpha^{\prime} k\right), \quad \forall \alpha^{\prime} \in \mathbb{R}, \forall k \in K .
$$

Hence, $x \perp_{F} K$.

Theorem 15. Let $K$ be a vector subspace of $X$. If $\pi\left(\widehat{K_{F}}\right)=$ $X^{n} / D_{K}$, then $K$ is $f$-simultaneously proximal, where $\pi$ is the canonical map $x \rightarrow x+D_{k}$.

Proof. Let $\pi\left(\widehat{K_{F}}\right)=X^{n} / D_{K}$ and $x=\left(x_{1}, x_{2}, \ldots, x_{n}\right) \in X^{n}$. Then, $x+D_{K}=y+D_{K}$ for some $y \in \widehat{K_{F}}$. Hence, $x-y=k_{0}$ for some $k_{0} \in D_{K}$. Thus, $x=y+k_{0} \in \widehat{K_{F}}+D_{k}$. Therefore, $\widehat{K_{F}}+$ $D_{k}=X^{n}$. By Theorem $15, K$ is $f$-simultaneously proximal.

\section{3. $f$-Simultaneous Approximation in Quotient Space}

Definition 16. Let $K$ and $M$ be two vector subspaces of $X$ such that $M$ is closed and $M \subset K$. Suppose that $f$ is a positive real-valued function defined on $X$. Then, a function $\widetilde{f}:(X / M)^{n} \rightarrow \mathbb{R}$ can be defined as follows:

$$
\begin{array}{r}
\tilde{f}\left(x_{1}+M, x_{2}+M, \ldots, x_{n}+M\right) \\
=\inf \left\{\sum_{i=1}^{n} f\left(x_{i}+y\right): y \in M\right\}
\end{array}
$$

for each $\left(x_{1}, x_{2}, \ldots, x_{n}\right) \in X^{n}$.

Theorem 17. Let $K$ and $M$ be two vector subspaces of $X$ such that $M \subset K$. If $k_{0}$ is a point of $f$-best simultaneous approximation to $\left(x_{1}, x_{2}, \ldots, x_{n}\right)$ in $K$, then $k_{0}+M$ is an $\tilde{f}$-best simultaneous approximation to $\left(x_{1}, x_{2}, \ldots, x_{n}\right)+M$ in $K / M$.

Proof. Suppose that $k_{0}+M$ is not $\tilde{f}$-best simultaneous approximation to $\left(x_{1}+M, x_{2}+M, \ldots, x_{n}+M\right)$ in $K / M$. Then,

$$
\tilde{f}\left(\left(x_{i}-k_{0}+M\right)_{i=1}^{n}\right) \not \tilde{f}\left(\left(x_{i}-k+M\right)_{i=1}^{n}\right)
$$

for at least $k \in K$, say $k_{1} \in K$, such that

$$
\tilde{f}\left(\left(x_{i}-k_{1}+M\right)_{i=1}^{n}\right)<\tilde{f}\left(\left(x_{i}-k_{0}+M\right)_{i=1}^{n}\right) .
$$

Since

$$
\begin{aligned}
\tilde{f}\left(\left(x_{i}-k_{0}+M\right)_{i=1}^{n}\right) & =\inf \left\{\sum_{i=1}^{n} f\left(x_{i}-k_{0}+y\right): y \in M\right\} \\
& \leq \sum_{i=1}^{n} f\left(x_{i}-k_{0}\right),
\end{aligned}
$$

we have

$$
\tilde{f}\left(\left(x_{i}-k_{1}+M\right)_{i=1}^{n}\right)<\sum_{i=1}^{n} f\left(x_{i}-k_{0}\right) .
$$

Thus, for some $m_{0} \in M$, we have

$$
\sum_{i=1}^{n} f\left(x_{i}-k_{1}+m_{0}\right)<\sum_{i=1}^{n} f\left(x_{i}-k_{0}\right)
$$

SO,

$$
\sum_{i=1}^{n} f\left(x_{i}-\left(k_{1}-m_{0}\right)\right)<\sum_{i=1}^{n} f\left(x_{i}-k_{0}\right) .
$$

Since $M \subset K$ implies that $k_{1}-m_{0} \in K$, therefore, $k_{0}$ is not $f$-best simultaneous approximation to $\left(x_{1}, x_{2}, \ldots, x_{n}\right)$ in $K$, which is a contradiction.

Corollary 18. Let $K$ and $M$ be two vector subspaces of $X$ such that $M \subset K$. Then, if $K$ is $f$-simultaneously proximal in $X$, then $K / M$ is $\widetilde{f}$-simultaneously proximal in $X / M$.

Proof. If $K$ is $f$-simultaneously proximal in $X$, then there exists at least $k_{0} \in K$ such that $k_{0}$ is $f$-best simultaneous approximation to $\left(x_{1}, x_{2}, \ldots, x_{n}\right)$ in $K$. Thus by Theorem 11, $k_{0}+M$ is an $\tilde{f}$-best simultaneous approximation to $\left(x_{1}, x_{2}, \ldots, x_{n}\right)+M$ in $K / M$, so, $K / M$ is $\widetilde{f}$-simultaneously proximal in $X / M$.

Theorem 19. Let $K$ and $M$ be two vector subspaces of $X$ such that $M \subset K$. If $M$ is $f$-simultaneously proximal in $X$ and $K / M$ is $\widetilde{f}$-simultaneously proximal in $X / M$, then $K$ is $f$ simultaneously proximal in $X$.

Proof. Since $K / M$ is $\widetilde{f}$-simultaneously proximal in $X / M$, then there exists $k_{0} \in K$ such that $k_{0}+M$ is $\tilde{f}$-best simultaneous approximation to $\left(x_{1}, x_{2}, \ldots, x_{n}\right)+M$ from $K / M$, so,

$$
\begin{aligned}
\tilde{f}\left(\left(x_{i}-k_{0}+M\right)_{i=1}^{n}\right) & \leq \tilde{f}\left(\left(x_{i}-k+M\right)_{i=1}^{n}\right), \quad \forall k \in K, \\
\Downarrow & \\
\tilde{f}\left(\left(x_{i}-k_{0}+M\right)_{i=1}^{n}\right) & =\inf _{m \in M} \sum_{i=1}^{n} f\left(x_{i}-k_{0}+m\right) \\
& \leq \inf _{m \in M} \sum_{i=1}^{n} f\left(x_{i}-k+m\right),
\end{aligned}
$$

for all $k \in K$. Note that

$$
\begin{aligned}
\inf _{m \in M} & \sum_{i=1}^{n} f\left(x_{i}-k_{0}+m\right) \\
& =F_{M}\left(x_{1}-k_{0}, x_{2}-k_{0}, \ldots, x_{n}-k_{0}\right) \\
& \leq F_{M}\left(x_{1}-k, x_{2}-k, \ldots, x_{n}-k\right) .
\end{aligned}
$$


Since $M$ is $f$-simultaneously proximal in $X$, then there exists $m_{0} \in M$ such that

$$
\begin{aligned}
F_{M} & \left(x_{1}-k_{0}, x_{2}-k_{0}, \ldots, x_{n}-k_{0}\right) \\
& =\sum_{i=1}^{n} f\left(x_{i}-k_{0}-m_{0}\right) \\
& \leq \sum_{i=1}^{n} f\left(x_{i}-k-m\right),
\end{aligned}
$$

for all $m \in M$ and $k \in K$. So,

$$
\sum_{i=1}^{n} f\left(x_{i}-\left(k_{0}+m_{0}\right)\right) \leq \sum_{i=1}^{n} f\left(x_{i}-(k+m)\right),
$$

for all $m \in M$ and $k \in K$. Hence,

$$
\begin{aligned}
& \sum_{i=1}^{n} f\left(x_{i}-\left(k_{0}+m_{0}\right)\right) \\
& \quad=\inf \left\{\sum_{i=1}^{n} f\left(x_{i}-(k+m)\right): m \in M, k \in K\right\} .
\end{aligned}
$$

So, $k_{0}+m_{0}$ is an $f$-best simultaneous approximation to $\left(x_{1}, x_{2}, \ldots, x_{n}\right)$ from $K$ and $K$ is $f$-simultaneously proximal in $X$.

Theorem 20. Let $K$ and $M$ be two vector subspaces of $X$ such that $M \subset K$. If $M$ is $f$-simultaneously proximal in $X$ and $K$ is $f$-simultaneously Chebyshev in $X$, then $K / M$ is $\tilde{f}$ simultaneously Chebyshev in $X / M$.

Proof. Suppose not, then there exists $\left(x_{1}, x_{2}, \ldots, x_{n}\right)+M \in$ $X / M$, and $k_{1}+M, k_{2}+M \in P_{K / M}^{\tilde{f}}\left(\left(x_{1}, x_{2}, \ldots, x_{n}\right)+M\right)$ such that $k_{1}+M \neq k_{2}+M$. Thus, $k_{1}-k_{2} \notin M$. Since $M$ is $f$ simultaneously proximal in $X$, then

$$
\begin{aligned}
& P_{M}^{f}\left(x_{1}-k_{1}, x_{2}-k_{1}, \ldots, x_{n}-k_{1}\right) \neq \phi, \\
& P_{M}^{f}\left(x_{1}-k_{2}, x_{2}-k_{2}, \ldots, x_{n}-k_{2}\right) \neq \phi .
\end{aligned}
$$

Let $m_{1} \in P_{M}^{f}\left(x_{1}-k_{1}, x_{2}-k_{1}, \ldots, x_{n}-k_{1}\right)$ and $m_{2} \in P_{M}^{f}\left(x_{1}-\right.$ $\left.k_{2}, x_{2}-k_{2}, \ldots, x_{n}-k_{2}\right)$. By Theorem 13, $k_{1}+m_{1}$ and $k_{2}+$ $m_{2}$ are $f$-best simultaneous approximation to $\left(x_{1}, x_{2}, \ldots, x_{n}\right)$ from $K$. Since $K$ is $f$-simultaneously Chebyshev in $X$, then $k_{1}+m_{1}=k_{2}+m_{2}$, and, hence, $k_{1}-k_{2}=m_{1}-m_{2} \in M$, which is a contradiction.

Theorem 21. Let $K$ and $M$ be two vector subspaces of $a$ topological vector space $X$. If $M$ is $f$-simultaneously Chebyshev in $X$, then the following assertions are equivalent:

(i) $K / M$ is $\tilde{f}$-simultaneously Chebyshev in $X / M$;

(ii) $K+M$ is simultaneously Chebyshev in $X$.

Proof. (i $\Rightarrow$ ii) By hypothesis, $(K+M) / M=K / M$ is $\tilde{f}$-simultaneous Chebyshev. Assume that $K+M$ is not $f$-simultaneous Chebyshev in $X$. Then, there exists $x=$ $\left(x_{1}, \ldots, x_{n}\right) \in X^{n}$ which has two distinct $f$-best simultaneous approximations, say $\ell_{0}$ and $\ell_{1} \in K+M$. Thus, we have $\ell_{0}$ and $\ell_{1} \in P_{K+M}^{f}(x)$. Since $M \subseteq K+M$, we have that $\ell_{0}+M$ and $\ell_{1}+M \in P_{(K+M) / M}^{f}(x+M)=P_{K / M}^{f}(x+M)$. By hypothesis, $K / M$ is $\tilde{f}$-simultaneous Chebyshev, and so $\ell_{0}+M=\ell_{1}+M$. Then, there exists $m_{0} \in M \backslash\{0\}$ such that $\ell_{1}=\ell_{0}+m_{0}$. Thus, we conclude that

$$
\begin{aligned}
& \sum_{i=1}^{n} f\left(\left(x_{i}-\ell_{0}\right)-m_{0}\right) \\
& \quad=\sum_{i=1}^{n} f\left(x_{i}-\ell_{1}\right) \\
& \quad=\inf _{m \in M}\left\{\sum_{i=1}^{n} f\left(x_{i}-\left(\ell_{0}+m\right)\right)\right\} \\
& \quad \leq\left\{\sum_{i=1}^{n} f\left(\left(x_{i}-\ell_{0}\right)-m\right)\right\}, \quad \forall m \in M \\
& =F_{M}\left(x-\ell_{0}\right) .
\end{aligned}
$$

So, $m_{0}$ and 0 are $f$-best simultaneous approximations to $x-\ell_{0}$ from $M$. Hence, $M$ is not $f$-simultaneously Chebyshev. This is a contradiction.

(ii $\Rightarrow$ i) Assume that (i) does not hold. Then, there exists $x+M \in K / M$ which has two distinct $\widetilde{f}$-best simultaneous approximations, say $k+M$ and $k^{\prime}+M \in K / M$; thus, $k-k^{\prime} \notin$ $M$. Since $M$ is $f$-simultaneously proximal, so there exist $f$ best simultaneous approximations $m$ and $m^{\prime}$ to $x-k$ and $x-k^{\prime}$ from $M$, respectively. Therefore, we have $m \in P_{M}^{f}(x-k)$ and $m^{\prime} \in P_{M}^{f}\left(x-k^{\prime}\right)$. Since $M \subseteq K+M, k+M$ and $k^{\prime}+M \in$ $P_{K / M}^{f}(x+M)=P_{(K+M) / M}^{f}(x+M)$, so $k+m$ and $k^{\prime}+m^{\prime} \epsilon$ $P_{K+M}^{f}(x)$. But $K+M$ is $f$-simultaneously Chebyshev. Thus we get $k+m=k^{\prime}+m^{\prime}$, and therefore $k-k^{\prime} \in M$. This is a contradiction.

Definition 22. A subset $K$ of $X$ is called $f$-quasisimultaneously Chebyshev if $P_{K}^{f}(x)$ is non-empty and $f$-compact set in $X$, for all $x=\left(x_{1}, x_{2}, \ldots, x_{n}\right) \in X^{n}$.

Theorem 23. Let $f$ be a positive function, $M$ an $f$-simultaneously proximal vector subspace of $X$, and $K f$-quasisimultaneously Chebyshev of $X$ such that $M \subset K$. Then, $K / M$ is $\tilde{f}$-quasi-simultaneously Chebyshev in $X^{n} / M$.

Proof. Since $K$ is $f$-simultaneously proximal in $X$, then by Corollary $12, K / M$ is $\tilde{f}$-simultaneously proximal in $X / M$. Let $x=\left(x_{1}, x_{2}, \ldots, x_{n}\right) \in X^{n}$ and $\left(k_{n}+M\right)$ a sequence in $P_{K / M}^{\tilde{f}}(x+M)$. For every $n$, there exists $m_{n} \in M$ such that $k_{n}+m_{n}=k_{n}^{\prime} \in P_{K}^{f}(x)$. But since $M$ is a vector subspace, we have

$$
k_{n}^{\prime}+M=k_{n}+m_{n}+M=k_{n}+M
$$


Since $K$ is $f$-quasi-simultaneously Chebyshev of $X$, the sequence $\left\{k_{n}\right\}$ has a subsequence $\left\{k_{n_{i}}\right\}$ which is $f$-convergent to $k_{0} \in P_{K}^{f}(x)$, meaning that

$$
f\left(k_{n_{i}}-k_{0}\right) \longrightarrow 0
$$

But

$$
\tilde{f}\left(k_{n_{i}}-k_{0}+M\right) \leq f\left(k_{n_{i}}-k_{0}\right) \longrightarrow 0 .
$$

Hence,

$$
\tilde{f}\left(k_{n_{i}}-k_{0}+M\right) \longrightarrow 0 .
$$

Consequently, $P_{K / M}^{\hat{f}}(x+M)$ is $\tilde{f}$-compact and $K / M$ is $\tilde{f}$-quasi-simultaneously Chebyshev. This completes the proof.

\section{Acknowledgments}

The authors would like to express their appreciation and gratitude to the editor and the anonymous referees for their comments and suggestions for this paper. Also, the authors wish to thank Professor Sharifa Al-Sharif for her valuable suggestions and comments.

\section{References}

[1] W. W. Breckner and B. Brosowski, "Ein Kriterium zur Charakterisierung von sonnen," Mathematica, vol. 13, pp. 181-188, 1971.

[2] T. D. Narang, "On $f$-best approximation in topological spaces," Archivum Mathematicum, vol. 21, no. 4, pp. 229-233, 1985.

[3] T. D. Narang, "Approximation relative to an ultra function," Archivum Mathematicum, vol. 22, no. 4, pp. 181-186, 1986.

[4] P. Govindarajulu and D. V. Pai, "On properties of sets related to $f$-projections," Journal of Mathematical Analysis and Applications, vol. 73, no. 2, pp. 457-465, 1980.

[5] D. V. Pai and P. Veermani, "Applications of fixed point theorems in optimization and best approximation," in Nonlinear Analysis and Application, S. P. Singh and J. H. Barry, Eds., pp. 393-400, Marcel Dekker, New York, NY, USA, 1982.

[6] M. R. Haddadi and J. Hamzenejad, "Best approximation in TVS," Caspian Journal of Mathematical Sciences, vol. 1, no. 2, pp. 75-70, 2012.

[7] S. V. R. Naidu, “On best simultaneous approximation," Publications de l'Institut Mathématique, vol. 52(66), pp. 77-85, 1992.

[8] F. B. Saidi, D. Hussein, and R. Khalil, "Best simultaneous approximation in $L^{p}(I, E)$," Journal of Approximation Theory, vol. 116, no. 2, pp. 369-379, 2002.

[9] M. Abrishami Moghaddam, "On $f$-best approximation in quotient topological vector spaces," International Mathematical Forum, vol. 5, no. 9-12, pp. 587-595, 2010. 


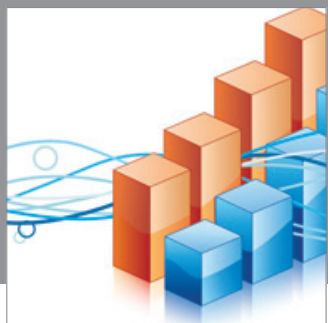

Advances in

Operations Research

mansans

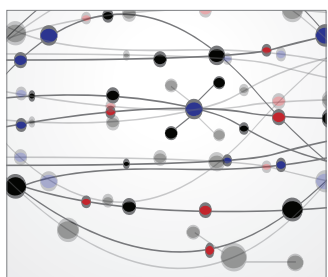

The Scientific World Journal
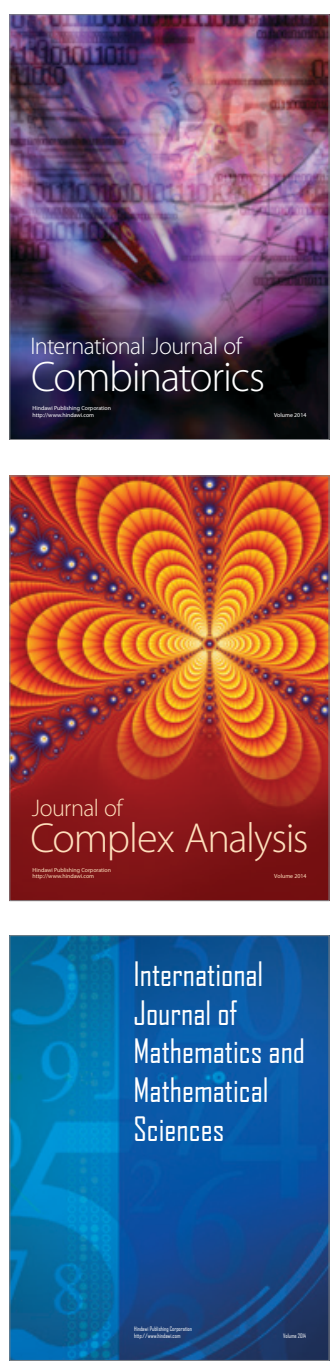
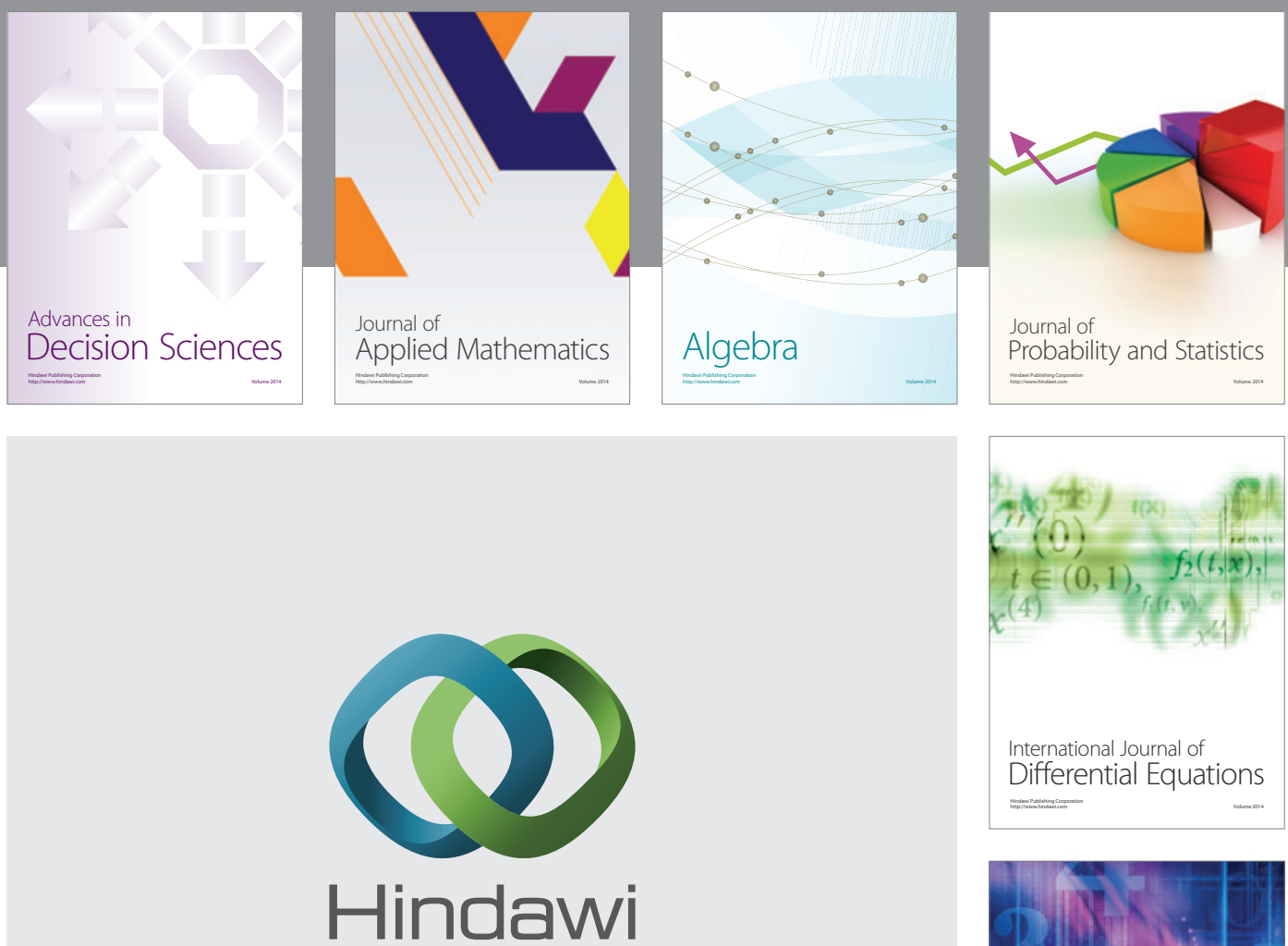

Submit your manuscripts at http://www.hindawi.com
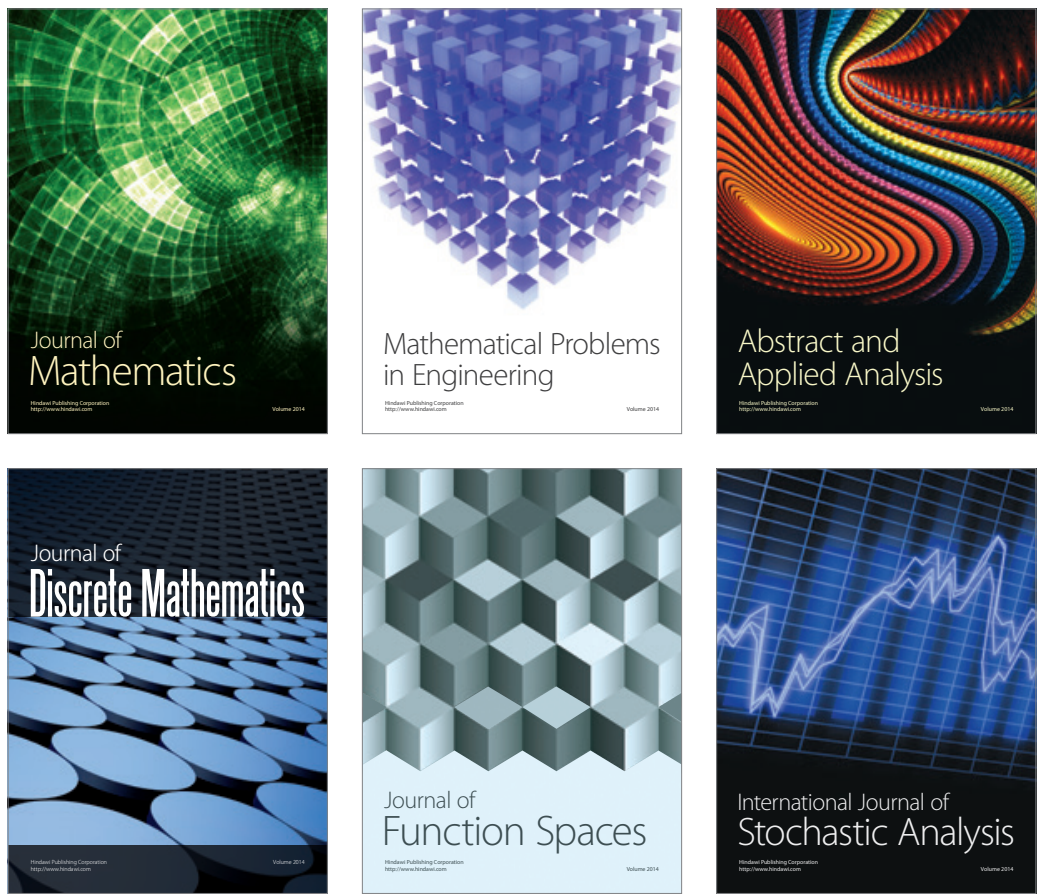

Journal of

Function Spaces

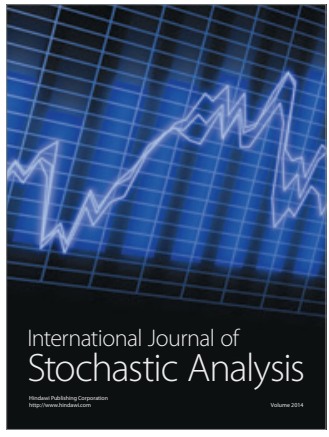

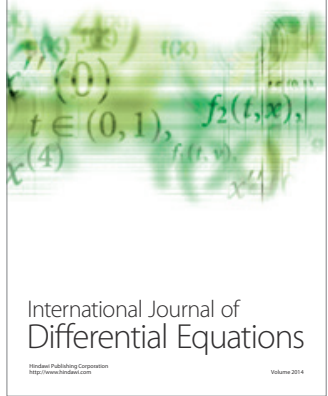
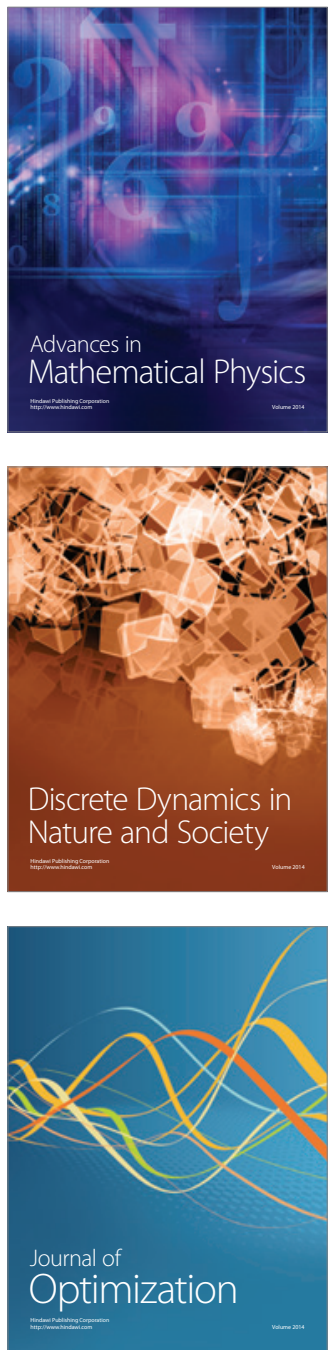